\title{
Monocytes and macrophages in chronic sarcoidosis pathology
}

\author{
To the Editor:
}

KAISER et al. [1] make a compelling case for T-lymphocytes in the pathogenesis of sarcoidosis, particularly when considering the initiation of granulomatous inflammation and patients with acute, self-resolving sarcoidosis (Lofgren's syndrome). However, in some situations it seems that T-cells are less important. For example, SCID (severe combined immunodeficient) mice, which have no lymphocytes, develop macrophage-rich granulomas when infected with mycobacteria [2]. In clinical practice, powerful suppression of T-lymphocytes with anti-rejection medication cannot prevent recurrence of granulomas in one third of patients who have undergone lung transplantation for pulmonary sarcoidosis [3].

Visually, chronic sarcoid granulomas have a well-defined macrophage-rich core and a sparse peripheral ring of lymphocytes. Indeed, pathologists use the term "naked granulomas" to contrast macrophage-dominant sarcoid pathology with the lymphocyte-rich lesions of tuberculosis. Recently, animal models and studies in patients have highlighted the importance of macrophages and their bone-marrow derived precursors, blood monocytes, in chronic sarcoidosis pathology. Mice genetically engineered with overactive mTORC1 specifically in their myeloid cells spontaneously developed granulomas in the lungs and skin reminiscent of sarcoidosis, and mTORC1 activation in biopsies correlated with disease progression in sarcoidosis patients [4]. Several clinical studies have demonstrated heightened inflammatory responses in circulating blood monocytes from sarcoidosis patients [5-7].

In sarcoidosis it is likely that there is a complex interplay between adaptive and innate immunity, represented by T-lymphocytes and monocyte-derived macrophages. An important research focus going forward will be to identify the drivers of non-resolving and progressive sarcoidosis that lead to considerable morbidity, loss of quality of life, and economic hardship.

@ERSpublications

Don't neglect monocytes and macrophages as drivers of chronic sarcoidosis http://bit.ly/2L2I5Yp

Cite this article as: Fraser SD, Hart SP. Monocytes and macrophages in chronic sarcoidosis pathology. Eur Respir J 2019; 54: 1901626 [https://doi.org/10.1183/13993003.01626-2019].

Simon D. Fraser and Simon P. Hart $\mathbb{D}$

Respiratory Research Group, Hull York Medical School, Castle Hill Hospital, Cottingham, UK.

Correspondence: Simon P. Hart, Hull York Medical School, Respiratory Research Group, Academic Respiratory Medicine, Castle Hill Hospital, Cottingham, HU16 5JQ, UK. E-mail: s.hart@hull.ac.uk

Received: 15 Aug 2019 | Accepted: 23 Aug 2019

Conflict of interest: S.D. Fraser reports grants from SarcoidosisUK/British Lung Foundation and Foundation for Sarcoidosis Research, outside the submitted work. S.P. Hart reports grants from SarcoidosisUK/British Lung Foundation and Foundation for Sarcoidosis Research, outside the submitted work.

\section{References}

1 Kaiser Y, Eklund A, Grunewald J. Moving target: shifting the focus to pulmonary sarcoidosis as an autoimmune spectrum disorder. Eur Respir J 2019; 54: 1802153.

2 Goldmann T, Zissel G, Sen Gupta R, et al. Formation of granulomas in the lungs of severe combined immunodeficient mice after infection with bacillus Calmette-Guerin. Am J Pathol 2001; 158: 1890-1891.

3 Schultz HH, Andersen CB, Steinbruuchel D, et al. Recurrence of sarcoid granulomas in lung transplant recipients is common and does not affect overall survival. Sarcoidosis Vasc Diffuse Lung Dis 2014; 31: 149-153.

4 Linke M, Pham HT, Katholnig K, et al. Chronic signaling via the metabolic checkpoint kinase mTORC1 induces macrophage granuloma formation and marks sarcoidosis progression. Nat Immunol 2017; 18: 293-302. 
5 Talreja J, Farshi P, Alazizi A, et al. RNA-sequencing identifies novel pathways in sarcoidosis monocytes. Sci Rep 2017; 7: 2720.

6 Fraser SD, Sadofsky LR, Kaye PM, et al. Reduced expression of monocyte CD200R is associated with enhanced proinflammatory cytokine production in sarcoidosis. Sci Rep 2016; 6: 38689.

7 Crawshaw A, Kendrick YR, McMichael AJ, et al. Abnormalities in iNKT cells are associated with impaired ability of monocytes to produce IL-10 and suppress T-cell proliferation in sarcoidosis. Eur J Immunol 2014; 44: $2165-2174$.

Copyright @ERS 2019 